\title{
Small cerebellar cortex volume in patients with active Cushing's syndrome
}

\author{
Alicia Santos ${ }^{1,+}$, Eugenia Resmini ${ }^{1}$, Iris Crespo ${ }^{1}$, Patricia Pires ${ }^{2}$, \\ Yolanda Vives-Gilabert ${ }^{2}$, Esther Granell ${ }^{3}$, Elena Valassi' ${ }^{1}$, Beatriz Gómez-Anson ${ }^{3}$, \\ M Antonia Martínez-Momblán ${ }^{1,4}$, Maria Mataró ${ }^{5}$ and Susan M Webb ${ }^{1}$ \\ ${ }^{1}$ Endocrinology/Medicine Departments, Hospital Sant Pau, Centro de Investigación Biomédica en Red \\ de Enfermedades Raras (CIBER-ER, Unidad 747), ISCIII, IIB-Sant Pau, Universitat Autònoma de Barcelona (UAB), \\ 08193 Bellaterra (Cerdanyola del Vallès), Spain, ${ }^{2}$ INNDACYT, Avenida Europa 20, Hospitalet de Llobregat, Barcelona, \\ Spain, ${ }^{3}$ Neuroradiology Unit, Hospital Sant Pau, and IIB-Sant Pau, Universitat Autònoma de Barcelona (UAB), \\ Barcelona, Spain, ${ }^{4}$ Escola Universitària d'Infermeria, Hospital Sant Pau. Universitat Autònoma de Barcelona (UAB), \\ Barcelona, Spain and ${ }^{5}$ Department of Psychiatry and Clinical Psychobiology, Psychology Faculty, Institute for Brain, \\ Cognition and Behaviour (IR3C), Universitat de Barcelona (UB), Barcelona, Spain \\ ${ }^{\dagger}$ A Santos is now at Department of Endocrinology, Hospital de la Santa Creu i Sant Pau, C/Sant Antoni Maria \\ Claret 167, 08025 Barcelona, Spain
}

Correspondence should be addressed to A Santos Email asantos@santpau.cat

\begin{abstract}
Objective: Cushing's syndrome (CS) is associated with neuropsychological deficits. As the cerebellum plays a key role in neuropsychological functions it may be affected in CS. The aim of this study was to investigate whether patients with CS have a smaller cerebellar volume than healthy controls, and to analyse whether cerebellar volume is associated with neuropsychological performance and clinical parameters.

Design: A cross-sectional study was performed.

Methods: Thirty-six CS patients (15 with active CS and 21 with CS in remission) and 36 controls matched for age, sex, and education underwent neuropsychological testing, quality of life assessment, clinical evaluation, and magnetic resonance imaging brain scan. Cerebellar volumes (white matter and cortex, bilateral) were calculated using FreeSurfer Software. Results: Patients with active CS showed smaller bilateral cerebellar cortex volumes than controls (left, $P=0.035$ and right, $P=0.034)$, as well as a trend toward smaller right cerebellar cortex volumes than patients in remission CS ( $P=0.051)$. No differences were observed in the volume of cerebellar white matter between the three groups. Both right and left cerebellar cortex volumes correlated negatively with triglyceride levels (right: $r=-0.358, P=0.002$ and left: $r=-0.317$, $P=0.005$ ) and age at diagnosis (right: $r=-0.433, P=0.008$ and left: $r=-0.457, P=0.005$ ). Left cerebellar cortex volume also correlated positively with visual memory performance $(r=0.245, P=0.038)$. Right cerebellar cortex volume positively correlated with quality-of-life scores $(r=0.468, P=0.004)$.

Conclusions: The cerebellar cortex volume is smaller in active CS patients than in controls. This finding is associated with poor visual memory and quality of life and is mostly pronounced in patients with higher triglyceride levels and older age at diagnosis.
\end{abstract}

\section{Introduction}

Cushing's syndrome (CS) is a rare disease caused by chronic glucocorticoid excess. It is characterized by central obesity, moon face, muscle weakness, red or purple striae, easy bruising, bone loss, hypertension, fatigue, lack of libido, emotional lability, and depression $(1,2,3)$. It has also been associated with cognitive impairment, particularly affecting memory and frontal functions $(4,5,6,7,8,9)$.
(C) 2014 European Society of Endocrinology Printed in Great Britain
Published by Bioscientifica Ltd. 
Chronic exposure to excess glucocorticoids can exert a neurotoxic effect $(10,11)$. In CS, this has been specifically associated with aging-like effects on the brain (12). Aging is associated with annual decreases in the volume of whole brain, the hippocampus, and the cerebellum $(13,14,15)$. Whole brain and hippocampal atrophy and neuropsychological dysfunction have been described in CS, but consensus is lacking about the reversibility of the effects of cortisol on the brain after biochemical control $(6,7,8,9$, $16,17,18,19)$. Furthermore, information regarding the cerebellum in CS is scarce. A 1971 study suggested that cerebellar atrophy was involved in CS, but no neuropsychological evaluation was performed and imaging techniques were not as precise as they are today (20).

The cerebellum has classically been associated with coordination, motor control, and muscle tone adjustments during movement to keep balance. However, it has long been suspected that the cerebellum has other roles, not only because it has multiple connections to cortical and subcortical brain regions, but also because more than half of the brain neurons are located in the cerebellum (21). Recently, the cerebellum has been related to emotional control and cognition, and linked to frontal/ executive functions, visuospacial skills, visual memory, verbal working memory, declarative and procedural memory, information processing speed, language, fluency, and emotional processing $(21,22,23)$. Cerebellar atrophy has been found in depression and in posttraumatic stress disorder, conditions in which hypercortisolism seems to have an important role (24). A reduction in cerebellar volumes has also been observed in patients with rheumatoid arthritis, a population that requires long-term glucocorticoid treatment (25). Taken together, these data suggest that hypercortisolism is involved in cerebellar volume reduction.

Our aim was to investigate whether patients with CS have a smaller cerebellar volume than healthy controls, and to analyse whether cerebellar volume is associated with neuropsychological performance, clinical parameters, and cortisol levels.

\section{Patients and methods}

\section{Patients}

CS patients who were routinely followed at Hospital de la Santa Creu i Sant Pau were recruited during their routine endocrinology visits. Healthy controls were donors from the blood donor service and individuals who had participated in previous studies at the center. All patients and controls gave signed informed consent to participate in the study, which was approved by the Hospital Ethics Committee.

Thirty-six CS patients (15 patients with active CS and 21 with CS in remission) and 36 matched controls were included in the study (Table 1). Each patient was matched to a control participant of the same sex, age ( \pm 3 years), and years of education ( \pm 3 years) to prevent the influence of age, sex, and education level. All patients and controls were right handed as the study involved brain magnetic resonance imaging (MRI; Edinburgh Handness Inventory >80) (26). Exclusion criteria for both CS patients and controls were as follows: age $>65$ years, growth hormone (GH) deficiency, history of drug or alcohol abuse, brain damage, and severe psychiatric or neurological illness. Diabetes mellitus was also considered as an exclusion criterion as it has been associated with a reduction in cerebellar volume (27). For controls, exclusion criteria additionally included history of endocrine disease or glucocorticoid exposure.

CS patients were considered in remission after surgery if they presented adrenal insufficiency or if morning cortisol suppression $(<50 \mathrm{nmol} / \mathrm{l})$ was observed after $1 \mathrm{mg}$ dexamethasone overnight (28), and if repeated

Table 1 Clinical characteristics of CS patients and controls.

\begin{tabular}{|c|c|}
\hline & CS in remission $(n=21)$ \\
\hline Age (years) & $41.9 \pm 10.4$ \\
\hline Sex (female/male) & $17 / 4$ \\
\hline Years of education & $13.1 \pm 3.4$ \\
\hline Origin of CS & $\begin{array}{l}18 \text { pituitary and } \\
\text { three adrenal }\end{array}$ \\
\hline $\begin{array}{l}\text { Duration of hyper- } \\
\text { cortisolism (months) }\end{array}$ & $61.8 \pm 32.2$ \\
\hline Delay to diagnosis (months) & $42.4 \pm 32.9$ \\
\hline Age at diagnosis & $34.9 \pm 9.3$ \\
\hline
\end{tabular}

\begin{tabular}{l}
\hline Active CS $(n=15)$ \\
\hline $44.2 \pm 9.3$ \\
$13 / 2$ \\
$13.7 \pm 2.7$ \\
Ten pituitary, three adrenal, \\
one AIMAH, and one ectopic \\
$62.2 \pm 59.1$ \\
$47.4 \pm 51.6$ \\
$40.2 \pm 9.6$
\end{tabular}

\begin{tabular}{|c|}
\hline Controls $(n=36)$ \\
\hline $42.7 \pm 9.9$ \\
\hline $30 / 6$ \\
\hline $13.4 \pm 3.5$ \\
\hline- \\
\hline- \\
\hline - \\
\hline- \\
\hline
\end{tabular}

CS, Cushing's syndrome; AIMAH, ACTH-independent macronodular adrenal hyperplasia. 
24-h urinary free cortisol measures were within the normal range ( $<280 \mathrm{nmol} / 24 \mathrm{~h}$ ). Patients who did not fulfill these criteria were considered active.

\section{Clinical interview, neuropsychological assessment, and biochemistry}

Patients and controls underwent a complete clinical interview that included demographic and clinical data, family and medical history, current medical treatment and blood pressure, height, weight, and waist circumference assessment. CS patients were also asked about the history of their disease. Information was completed with data collection from their clinical files.

Duration of hypercortisolism was estimated as the time from symptom onset (in months) to the date of hypercortisolism remission (or current date in active patients), as previously described (9). The time from symptom onset was estimated through a detailed patient interview and from review of clinical notes and photographs. Delay to diagnosis was considered as the time from symptom onset (in months) to the date of diagnosis of CS.

Both patients and controls performed a battery of neuropsychological tests related to cerebellar functions.
Only total scores were included in the analysis, except for the Wisconsin Card Sorting Test (WCST), where mean time and perseverative errors were recorded. We specifically used the Grooved Pegboard (dominant and non-dominant hand) and the Trail Making Test A to evaluate motor functions, the Object Assembly and Block Design from WAIS-III for visuoconstructive functions, the Boston Naming Test and Vocabulary from WAIS-III for language, the Rey-Osterrieth Complex Figure for visual memory, the Symbol Digit Modality Test and WCST (mean time) for information processing speed, and Animals, FAS, WCST (perseverative errors), Digit Span Backwards and Trail Making Test B for executive functions. Table 2 provides further information about each test. Participants also performed two complementary questionnaires to assess depression (BDI-II) and anxiety (STAI), both the actual state (STAI State) and the personality trait (STAI Trait).

CS patients also completed a disease-specific qualityof-life questionnaire, the Cushing QoL (29). All neuropsychological assessments were performed by the same neuropsychologist (A Santos) to avoid inter-examiner variability.

The participants underwent blood and urine tests. We used a 24-h collection to assess urinary free cortisol using

Table 2 Description of the neuropsychological tests performed (in alphabetical order).

\begin{tabular}{lll}
\hline 1 & Test's name \\
\cline { 1 - 1 } 2 & $\begin{array}{l}\text { Animals } \\
\text { Block Design (from Wechsler } \\
\text { Adult Intelligence Scale } \\
\text { (WAIS-III)) }\end{array}$ \\
3 & $\begin{array}{c}\text { Boston Naming Test } \\
\text { (abbreviated version) }\end{array}$ \\
4 & $\begin{array}{c}\text { Digit Span Backwards } \\
\text { (from WAIS-III) }\end{array}$ \\
5 & $\begin{array}{c}\text { FAS } \\
6\end{array}$ \\
7 & $\begin{array}{c}\text { Grooved Pegboard } \\
\text { Object Assembly (from WAIS-III) }\end{array}$ \\
9 & $\begin{array}{c}\text { Rey-Osterrieth Complex Figure } \\
\text { (ROCF) }\end{array}$ \\
9 & $\begin{array}{c}\text { Symbol Digit Modality Test } \\
\text { (SDMT) }\end{array}$
\end{tabular}

10

11

12

Trail Making Test B (TMTB)

Vocabulary (from WAIS-III)

Wisconsin Card Sorting Test (WCST)

\section{Description}

A test that measures semantic fluency. Patients have to enumerate all the animals they can recall in $1 \mathrm{~min}$

This task evaluates visuospacial skills. Patients have to build figures following a model in a picture card. They have to use cubes with different colored sides (white, red or half white, and half red)

A language test that measures denomination. Patients are asked to name the pictures they are shown

This test measures working memory. The patients have to repeat a series of numbers backwards (i.e. '719' would be '917')

This test assesses phonetic fluency. Patients have to enumerate all the words they can in $1 \mathrm{~min}$. Words must begin with a specific letter (i.e. ' $\mathrm{F}$ ')

A test that measures fine motor skills (coordination and motor speed). Patients have to insert some pegs in a pegboard, as quick as possible. They will do it with both the dominant (DH) and non-dominant hand (NDH)

This test measures visuospatial skills. It consists in assembling a series of puzzles, as quickly as possible

A test that assesses visual memory. Patients have to copy a figure looking to a model and then draw it again without the model. They will also have to draw it again after $20 \mathrm{~min}$

A test that measures information processing speed, divided attention, visual scanning, and tracking. The patients see a model were symbols correspond to numbers. A sequence of symbols is shown. Using the model, patients will have to say the numbers that correspond to symbols as quickly as possible

This test measures divided attention. The patients have to connect numbers and letters in ascending order (i.e. 1-a-2-b-3-c)

A test that assesses language. The patient has to say the meaning of different words

A computerized test that measures cognitive flexibility. Four model cards are shown to patients. They have to match their cards with one of the models, trying to find the correct criteria (i.e. color). Criteria change over time 
a commercial RIA. Standard assay methods were used to assess cholesterol, triglyceride, and glucose levels from blood samples.

\section{MRI and cerebellar volumes}

MRI was obtained using a 3-Tesla Philips Achieva scanner (software version 2.1.3.2) and a specific acquisition protocol: 3DMPRAGE whole-brain sequence (repetition time $=6.7 \mathrm{~ms}$; echo time $=3.1 \mathrm{~ms}, 170$ slices; and voxel size $=0.889 \times 8.889 \times 1.2)$.

All images were postprocessed by the Port d'Informació Científica (PIC) at the Universitat Autònoma de Barcelona using the FreeSurfer Software (http://surfer.nmr.mgh. harvard.edu/). The volumes of right and left white matter and right and left cortex were obtained. The total cerebellar volume was calculated by adding up all these volumes.

Volumetric segmentation was performed automatically using FreeSurfer version 4.3.1 Image Analysis Software (http://surfer.nmr.mgh.harvard.edu/). This software is composed of $170 \mathrm{HP}$ blades with two quad-cores CPU (Hewlett Packard), each one with 16 GB of RAM, running over Scientific Linux version 5 (https://www.scientificlinux.org/). FreeSurfer processing includes motion correction, removal of non-brain tissue using a hybrid watershed/surface deformation procedure (30), automated Talairach transformation, and segmentation of the subcortical white matter and deep grey matter volumetric structures $(31,32)$. Postprocessing was launched using the PICNIC tool (https://neuroweb.pic.es). Both the automated image processing and the visual check were done by a single, blinded investigator.

All volumetric scores where normalized to the estimated intracranial volume of each individual, as previously described (9).

\section{Statistical analysis}

Statistical analysis was performed using IBM SPSS 21 Software (SPSS, Inc.). Normal distribution was analyzed using the Kolmogorov-Smirnov test. Comparisons between groups were performed using ANOVA followed by a Bonferroni's correction. Differences were considered significant when $P<0.05$. The $\chi^{2}$ test was used to compare categorical variables, and correlations were assessed using Pearson's coefficient. Data are reported as mean \pm s.D.

Direct scores of the tests were transformed into $Z$-scores to obtain a general score for each cognitive function. The general scores for each cognitive function were obtained by calculating the mean between the $Z$-scores of the tests included for each cognitive function. Tests in which higher scores were not related to a better performance were inverted.

\section{Results}

No differences were found between groups for age, sex, or education level. Table 1 summarizes clinical and demographic characteristics of CS patients and controls.

Patients in remission included 21 CS patients (17 females) who achieved cure of their hypercortisolism after treatment: 18 were of pituitary origin and three of adrenal origin. At the time of the study, six were on hydrocortisone therapy for adrenal insufficiency after surgery, five were taking antidepressants, and five had had previous radiotherapy.

Active patients included 15 CS patients (13 females) with active hypercortisolism: ten of pituitary origin, three of adrenal origin, one ectopic adrenocorticotropic hormone (ACTH) secretion of unknown origin, and one ACTH-independent macronodular adrenal hyperplasia (AIMAH). Fourteen of the 15 active patients were taking medication: 11 were on ketoconazole or metyrapone treatment, one was taking cabergoline, one was taking losartan treatment (a patient with AIMAH who responded to angiotensin receptor antagonists), and one was taking antidepressants.

Total cerebellar volume was calculated. Both grey matter (cortex) and white matter were analyzed separately

Table 3 Mean cerebellar volumes in patients and controls.

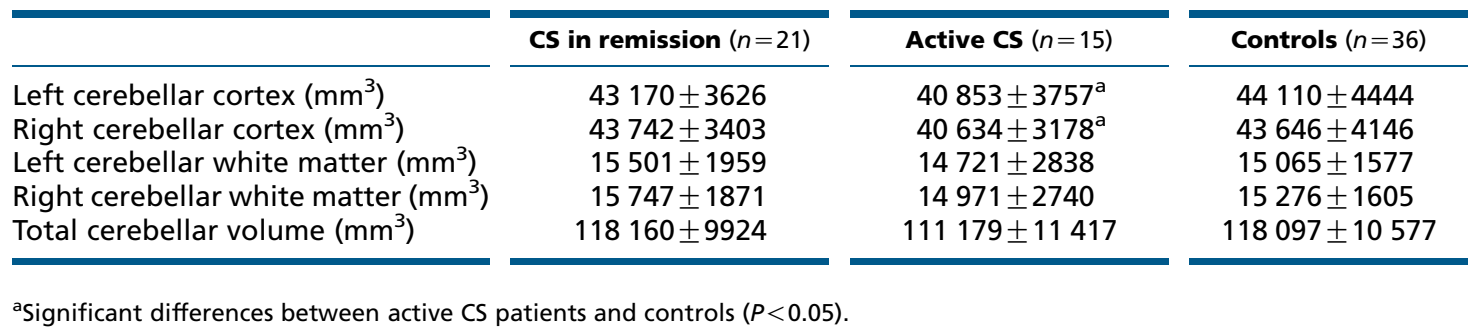



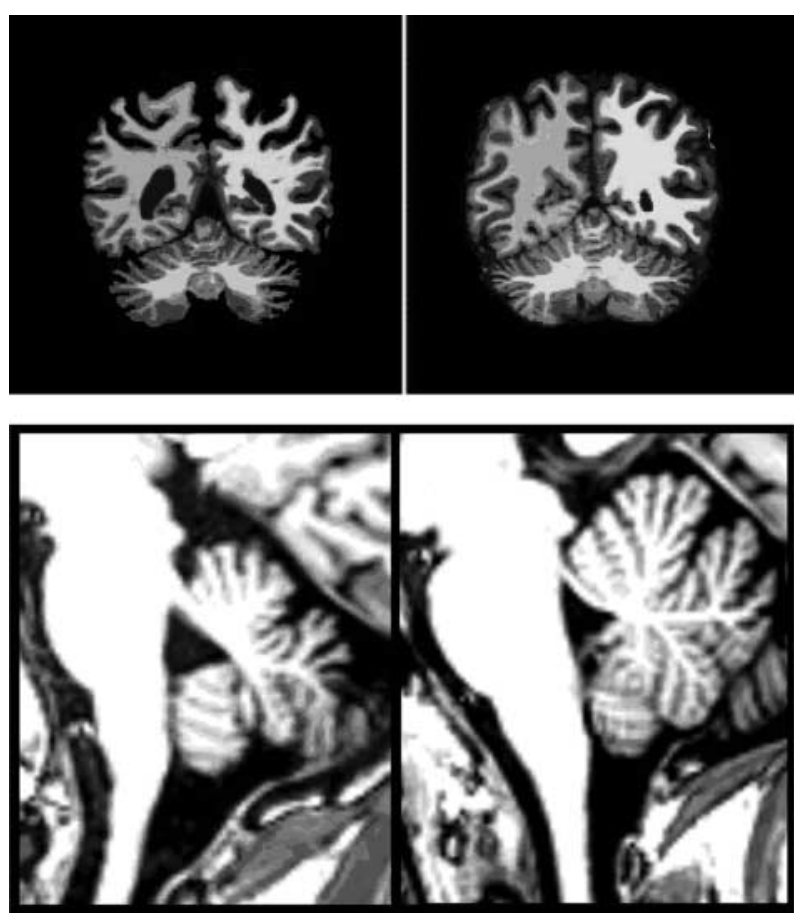

\section{Figure 1}

Images obtained from FreeSurfer and MRI (T1), corresponding to an active CS patient (left) and its matched healthy control (right). Reduction in cerebellar volume can be observed in the CS patient.

for each cerebellar hemisphere. Table 3 shows mean cerebellar volumes.

No differences were found between groups for total cerebellar volumes. However, in patients with active CS, cerebellar cortex volumes were smaller than those in controls (left, $P=0.035$ and right, $P=0.034$ ). The results did not change when the patient taking antidepressants and the corresponding healthy control were excluded from the analysis (left, $P=0.046$ and right, $P=0.032$ ). In contrast, cerebellar cortex volumes in CS patients in remission did not differ from those in controls. When the two CS patient groups were compared, active patients also tended to have a smaller right cerebellar cortex volume than patients in remission $(P=0.051)$. No differences were found in cerebellar white matter volumes between the three groups. Figure 1 shows an example of the MRI of a patient and her matched control.

Regarding neuropsychological functions, patients with active CS had a poorer performance in visual memory than controls $(P=0.006)$, and a tendency for poorer performance when compared with patients in remission $(P=0.066)$. Visual memory correlated with left cerebellar cortex volumes $(r=0.245, P=0.038)$. No difference between groups was found for other functions (fine motor skills, visuoconstructive function, language, information processing speed, or executive function; Fig. 2). Table 4 gives mean and s.D.s of each neuropsychological test. Furthermore, both patient groups had more depression (both $P<0.001$ ) and anxiety scores than controls (STAI State, $P=0.014$ for active CS and $P=0.024$ for patients in remission; STAI Trait, $P<0.001$ for both patient groups), although no difference was found between patients with active disease or those in remission. No correlation was found between these scores and cerebellar volumes.

Regarding clinical parameters, active CS patients had higher urinary free cortisol $(P<0.001)$, triglycerides $(P=0.010)$, waist circumference $(P=0.001)$, systolic blood pressure $(P=0.006)$, and diastolic blood pressure $(P<0.001)$ than controls. CS patients in remission showed higher waist circumference $(P=0.031)$, systolic blood pressure $(P=0.002)$, and diastolic blood pressure $(P<0.001)$ than controls. They also showed a tendency to higher triglycerides $(P=0.062)$. When the whole sample was analyzed, only triglycerides correlated negatively with right and left cerebellar cortex volumes (right: $r=-0.358$, $P=0.002$ and left: $r=-0.317, P=0.005)$. Both right and

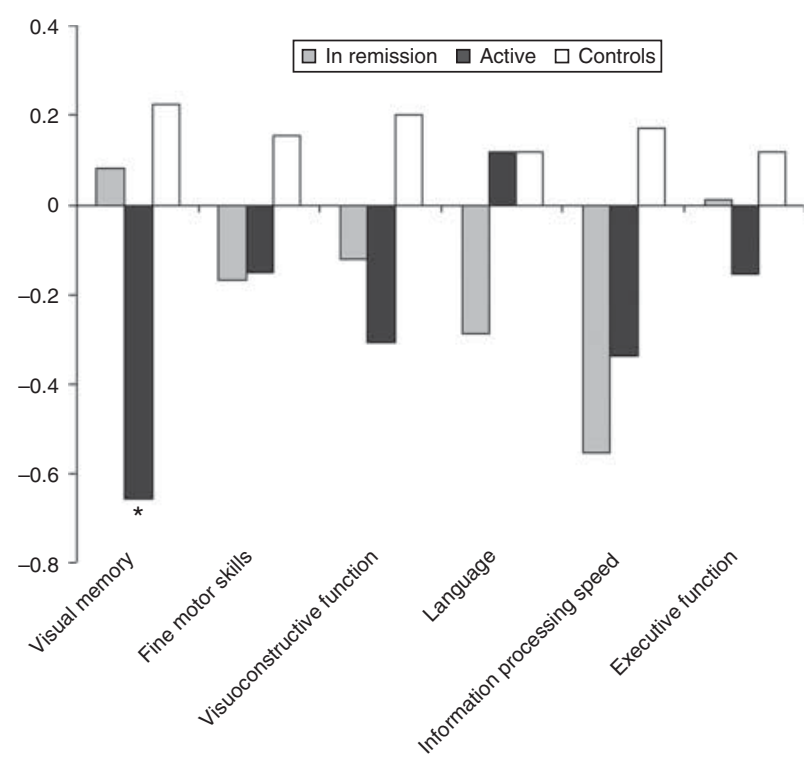

Figure 2

Z-scores of each cognitive function in CS patients (active and in remission) and controls, obtained from neuropsychological testing. *Significant differences between active CS patients and controls $(P=0.006)$. 
Table 4 Mean and SDS of neuropsychological tests.

\begin{tabular}{l}
\hline \\
\\
\hline Visual memory \\
ROCF immediate \\
ROCF delayed \\
Fine motor skills \\
Grooved DH ${ }^{\text {a }}$ \\
Grooved NDH \\
Visuoconstructive \\
Object Assembly \\
Block Design \\
Language \\
Vocabulary \\
Boston Naming Test \\
Information processing \\
speed \\
Symbol Digit \\
WCST (mean time) \\
Executive \\
WCST (perseverative \\
errors) \\
TMTB \\
Digits Backwards \\
FAS \\
Animals \\
\hline
\end{tabular}

\begin{tabular}{c} 
\\
CS in remission \\
\hline \\
$21.3 \pm 6.5$ \\
$20.8 \pm 6.4$ \\
$71.0 \pm 11.4$ \\
$72.8 \pm 10.5$ \\
$28.8 \pm 9.1$ \\
$40.2 \pm 21.1$ \\
$39.7 \pm 9.2$ \\
$12.8 \pm 1.3$ \\
$62.6 \pm 10.8$ \\
$2281.4 \pm 927.1$ \\
$10.4 \pm 9.9$ \\
$58.6 \pm 17.5$ \\
$5.5 \pm 1.5$ \\
$42.9 \pm 9.4$ \\
$26.2 \pm 4.9$ \\
\hline
\end{tabular}
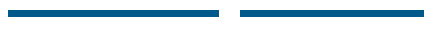

\begin{tabular}{|c|c|c|}
\hline \multicolumn{3}{|c|}{$P$ value } \\
\hline $\begin{array}{l}\text { CS in remission } \\
\text { vs controls }\end{array}$ & $\begin{array}{l}\text { Active CS } \\
\text { vs controls }\end{array}$ & $\begin{array}{c}\text { CS in remission } \\
\text { vs active }\end{array}$ \\
\hline 1.000 & 0.028 & 0.086 \\
\hline 1.000 & 0.004 & 0.062 \\
\hline 0.057 & 0.285 & 1.000 \\
\hline 1.000 & 1.000 & 1.000 \\
\hline 0.649 & 0.191 & 1.000 \\
\hline 0.808 & 0.449 & 1.000 \\
\hline 0.429 & 1.000 & 0.490 \\
\hline 0.446 & 1.000 & 0.987 \\
\hline
\end{tabular}

ROCF, Rey-Osterrieth Complex Figure; DH, dominant hand; NDH, non-dominant hand; WCST, Wisconsin Card Sorting Test; TMTB, Trail Making Test B. Total scores are given except when indicated in brackets.

${ }^{\mathrm{a}}$ For these tests higher scores indicate poorer performance (Grooved DH, Grooved NDH, WCST mean time, WCST perseverative errors, and TMTB).

left cerebellar cortex volumes were negatively correlated to age at diagnosis (right: $r=-0.433, P=0.008$ and left: $r=-0.457, P=0.005)$. Current age did not correlate with cerebellar volumes in active CS or patients in remission, but a correlation was found in the control group (right: $r=-0.426, P=0.010$ and left: $r=-0.559, P=0.000$ ).

The volume of the right cerebellar cortex correlated positively with the Cushing QoL quality-of-life scores $(r=0.468, P=0.004)$. No correlations were found for cerebellar volumes (white matter and cortex), levels of cholesterol, glucose and urinary free cortisol, duration of hypercortisolism, or delay to diagnosis.

\section{Discussion}

CS is associated with a decrease in whole brain and hippocampal volumes, although its reversibility after biochemical control of hypercortisolism is still a matter of debate $(9,16,18,19,33)$. In this study, we found that the cerebellar cortex (grey matter) was smaller in patients with active hypercortisolism than in control individuals. Glucocorticoids have been linked to cell death in brain regions such as the cerebellum and hippocampus (34).
They are also known to impair neurogenesis, present in both these structures (35). Glucocorticoids and stress can also modify dendritic structure, reducing synapsis and dendritic atrophy (dendritic simplification and retraction) $(36,37)$. As dendrites are part of the cortex, our results could also be explained by a reduction in dentrites induced by glucocorticoids.

In contrast with these findings in active patients, the cerebellar cortex was not smaller in CS patients in remission. Based on these data, we can speculate that hypercortisolemia leads to cerebellar shrinkage, which may be partially reversible after cure. Several studies have found that hippocampal volume increases after cure, so it is feasible that other brain structures may also improve after remission $(16,19)$. Our results are in line with a recent study in CS patients in remission that did not find the volume of cerebellar grey matter was smaller. In fact, the authors reported that the left posterior lobe of the cerebellum was larger (33). They suggested that neuronal reorganization after chronic stress could lead to dendrite atrophy in parts of the brain or to dendritic hypertrophy in others. This could explain the larger cerebellar volume that they found in contrast with smaller volumes in 
other structures. Following this hypothesis, even if dendritic atrophy occurred during the active phase of the disease, it could be compensated by a dendritic hypertrophy after biochemical cure. Longitudinal studies would be necessary to confirm these hypotheses.

In this study, smaller cerebellar cortex volumes were associated with several clinical variables. First, age at diagnosis correlated negatively with cerebellar volume, meaning that the volume of cerebellar cortex was smaller in patients who were diagnosed at an older age. Current age did not correlate with cerebellar volumes in active patients or in patients in remission, so the correlation with age at diagnosis may be related to other factors, such as exposure to hypercortisolism. The capacity for neurogenesis decreases significantly throughout life (38). Exposure to glucocorticoids at a later age may therefore be related to an increased risk of cerebellar reduction, as the capacity of neurons to regenerate is reduced with aging.

Second, we found a negative correlation between triglyceride levels and the volume of the cerebellar cortex, meaning that elevated triglycerides were related to a smaller cerebellar cortex volume. This is consistent with a study in which rats fed a medium-chain triglyceride supplemented diet had an aging-like effect in the cerebellar cortex, leading to a lower number of synapsis and synaptic mitochondria (39). The authors claimed that this diet could have aging or anti-aging effects, depending on the neuronal vulnerability of the cells, and the cerebellar cortex is particularly vulnerable to age. Therefore, high triglyceride levels, together with cortisol exposure, could have an aging-like effect in the cerebellar cortex in CS patients, leading to a volumetric reduction. Control of triglyceride levels may be helpful to avoid cerebellar impairment. However, further studies on dyslipidemia and cerebellar function are needed.

Third, quality of life, evaluated with the diseasespecific Cushing QoL questionnaire, correlated directly with right cerebellar cortex volume. This relation between quality of life and cerebellar cortex has not been reported previously, but since the cerebellum plays a role in the modulation of emotional responses (23), it may also be involved in self-perceived quality of life. In patients with right cerebellar insults, lesion size has been correlated with the severity of depression (40). Indeed, the cerebellum has been associated with emotional processing. Although we did not find any correlation between depression and cerebellar cortex, it is feasible that there may be a certain degree of lateralization for emotional processing, mood, and depression in the cerebellum.

Regarding neuropsychological results, visual memory performance correlated with left cerebellar cortex volume.
Even if memory has classically been related to the hippocampus, the cerebellum also seems to play a role in visual memory $(21,23)$. Specifically, visuospatial memory has been associated with the left-superior-posterior lobe of the cerebellum in both children with early deprivation and normal children (41). Our data show that patients with smaller left cerebellar volumes have poorer visual memory skills. This could lead to problems such as difficulties in remembering information from maps, or losing one's way in unknown places. These findings may have implications during the diagnostic process because neuropsychological impairment may be present. Apart from a neuropsychological evaluation, a detailed neurological examination may be helpful to identify further impairment.

It is also of note that patients in remission showed poorer neuropsychological performance than active patients in language and information processing speed, although this difference was not statistically significant. CS predisposes to chronic inflammation and cerebrovascular disease, even after cure (42). Long-term inflammation has been associated with impaired information processing speed (43), while cerebrovascular disease can lead to vascular dementia. Interestingly, in patients with vascular dementia, language function progressively declines (44). Patients in remission have been exposed to inflammation and cerebrovascular risk for a longer time than active patients, which may explain their worse performance. As language tends to be more preserved than information processing speed with normal aging (45), it is conceivable that newly diagnosed patients with active hypercortisolism still maintain normal language function.

A smaller cerebellar volume may have other clinical implications. As this portion of the brain is related to motor control and gait, a smaller volume might account for poor postural control and balance deficits in these patients $(46,47)$. These deficits may be even more severe in patients with obesity, a symptom also related to impaired balance and postural control (48) and to more falls than in normal-weight subjects (49). The risk of falls should be kept in mind in patients with CS as they have a higher incidence of osteoporosis and therefore a higher risk of fracture. The risk of falls should be specially considered in older patients, as it has been associated with higher morbidity, greater use of health care resources, and even a higher mortality (50). It would be interesting in the future to prospectively study the relationship between cerebellar volume and the number of falls.

This study has several limitations. The first one is the small sample size and the heterogeneity of the sample, 
challenges inherent to a rare disease such as CS. The number of patients included in the study was further reduced by the strict exclusion criteria. However, this restriction avoided the influence of factors which could affect cerebellar volume, such as diabetes mellitus, GH deficiency, older age, brain damage, history of drug or alcohol abuse, and severe psychiatric or neurological illnesses. The second limitation is that FreeSurfer is an automatic technique and it may not be as accurate as manual segmentation. It is feasible that measurement noise may have masked clearer correlations with clinical and neuropsychological parameters. A further limitation is that we did not perform a standard neurological evaluation, and such a study might have identified subtle cerebellar dysfunction in these CS patients. A final potential weakness is the cross-sectional design of the study. Longitudinal studies could also confirm whether the cerebellar cortex volume in active patients increases after cure, and if so, how long this takes to occur. Studies analysing the possible benefits of neuropsychological rehabilitation could also lead to improvements in QoL and neuropsychological functions.

In conclusion, we found that patients with active CS have a smaller cerebellar cortex, associated with poorer visual memory, decreased QoL, higher blood triglyceride levels, and older age at diagnosis. The absence of this volumetric reduction in patients in remission is encouraging, suggesting that it may be reversible, at least in part, after cortisol normalization.

\section{Declaration of interest}

The authors declare that there is no conflict of interest that could be perceived as prejudicing the impartiality of the research reported.

\section{Funding}

This work was supported by the Instituto de Salud Carlos III, the Spanish Ministry of Science and Innovation (MICINN, FIS080302), the European Commission (ERCUSYN PHP800200), and AGAUR (2014 SGR 355).

\section{Acknowledgements}

The authors thank all the patients and controls who participated in the study.

\section{References}

1 Arnaldi G, Angeli A, Atkinson AB, Bertagna X, Cavagnini F, Chrousos GP, Fava GA, Findling JW, Gaillard RC, Grossman AB et al. Diagnosis and complications of Cushing's syndrome: a consensus statement. Journal of Clinical Endocrinology and Metabolism $2003 \mathbf{8 8}$ 5593-5602. (doi:10.1210/jc.2003-030871)

2 Nieman LK, Biller BM, Findling JW, Newell-Price J, Savage MO, Stewart PM \& Montori VM. The diagnosis of Cushing's syndrome: an Endocrine Society clinical practice guideline. Journal of Clinical Endocrinology and Metabolism 200893 1526-1540. (doi:10.1210/jc.2008-0125)

3 Starkman MN, Schteingart DE \& Schork MA. Depressed mood and other psychiatric manifestations of Cushing's syndrome: relationship to hormone levels. Psychosomatic Medicine 198143 3-18. (doi:10.1097/ 00006842-198102000-00002)

4 Starkman MN, Giordani B, Berent S, Schork MA \& Schteingart DE. Elevated cortisol levels in Cushing's disease are associated with cognitive decrements. Psychosomatic Medicine 200163 985-993. (doi:10.1097/00006842-200111000-00018)

5 León-Carrión J, Atutxa AM, Mangas MA, Soto-Moreno A, Pumar A, Leon-Justel A, Martín-Rodriguez JF, Venegas E, Domínguez-Morales MR $\&$ Leal-Cerro A. A clinical profile of memory impairment in humans due to endogenous glucocorticoid excess. Clinical Endocrinology 200970 192-200. (doi:10.1111/j.1365-2265.2008.03355.x)

6 Dorn LD \& Cerrone P. Cognitive function in patients with Cushing's syndrome: a longitudinal perspective. Clinical Nursing Research 20009 420-440. (doi:10.1177/10547730022158672)

7 Forget H, Lacroix A \& Cohen H. Persistent cognitive impairment following surgical treatment of Cushing's syndrome. Psychoneuroendocrinology 200227 367-383. (doi:10.1016/S0306-4530(01)00059-2)

8 Tiemensma J, Kokshoorn NE, Biermasz NR, Keijser BJ, Wassenaar MJ, Middelkoop HA, Pereira AM \& Romijn JA. Subtle cognitive impairments in patients with long-term cure of Cushing's disease. Journal of Clinical Endocrinology and Metabolism 201095 2699-2714. (doi:10.1210/ jc.2009-2032)

9 Resmini E, Santos A, Gómez-Anson B, Vives Y, Pires P, Crespo I, Portella MJ, de Juan-Delago M, Barahona MJ \& Webb SM. Verbal and visual memory performance and hippocampal volumes, measured by 3-Tesla magnetic resonance imaging, in patients with Cushing's syndrome. Journal of Clinical Endocrinology and Metabolism 201297 663-671. (doi:10.1210/jc.2011-2231)

10 Joëls M, Karst H, Krugers HJ \& Lucassen PJ. Chronic stress: implications for neural morphology, function and neurogenesis. Frontiers in Neuroendocrinology 200728 72-96. (doi:10.1016/j.yfrne. 2007.04.001)

11 McEwen BS. Physiology and neurobiology of stress and adaptation: central role of the brain. Physiological Reviews 200787 873-904. (doi:10.1152/physrev.00041.2006)

12 Michaud K, Forget H \& Cohen H. Chronic glucocorticoid hypersecretion in Cushing's syndrome exacerbates cognitive aging. Brain and Cognition 200971 1-8. (doi:10.1016/j.bandc.2009.02.013)

13 Enzinger C, Fazekas F, Matthews PM, Ropele S, Schmidt H, Smith S \& Schmidt R. Risk factors for progression of brain atrophy in aging: six-year follow-up of normal subjects. Neurology 200564 1704-1711. (doi:10.1212/01.WNL.0000161871.83614.BB)

14 Scahill RI, Frost C, Jenkins R, Whitwell JL, Rossor MN \& Fox NC. A longitudinal study of brain volume changes in normal aging using serial registered magnetic resonance imaging. Archives of Neurology 2003 60 989-994. (doi:10.1001/archneur.60.7.989)

15 Raz N, Lindenberger U, Rodrigue KM, Kennedy KM, Head D, Williamson A, Dahle C, Gerstorf D \& Acker JD. Regional brain changes in aging healthy adults: general trends, individual differences and modifiers. Cerebral Cortex 200515 1676-1689. (doi:10.1093/cercor/bhi044)

16 Bourdeau I, Bard C, Noël B, Leclerc I, Cordeau MP, Bélair M, Lesage J, Lafontaine L \& Lacroix A. Loss of brain volume in endogenous Cushing's syndrome and its reversibility after correction of hypercortisolism. Journal of Clinical Endocrinology and Metabolism 200287 1949-1954. (doi:10.1210/jcem.87.5.8493)

17 Simmons NE, Do HM, Lipper MH \& Laws ER. Cerebral atrophy in Cushing's disease. Surgical Neurology 200053 72-76. (doi:10.1016/ S0090-3019(99)00197-4) 
18 Gnjidić Z, Sajko T, Kudelić N, Malenica M, Vizner B, Vrkljan M, Hat J \& Rumboldt Z. Reversible "brain atrophy" in patients with Cushing's disease. Collegium Antropologicum 200832 1165-1170.

19 Starkman MN, Giordani B, Gebarski SS, Berent S, Schork MA \& Schteingart DE. Decrease in cortisol reverses human hippocampal atrophy following treatment of Cushing's disease. Biological Psychiatry 199946 1595-1602. (doi:10.1016/S0006-3223(99)00203-6)

20 Momose KJ, Kjellberg RN \& Kliman B. High incidence of cortical atrophy of the cerebral and cerebellar hemispheres in Cushing's disease. Radiology 197199 341-348. (doi:10.1148/99.2.341)

21 Tirapu-Ustarroz J, Luna-Lario P, Iglesias-Fernandez MD \& HernaezGoni P. Cerebellar contribution to cognitive process: current advances. Revue Neurologique 201153 301-315.

22 Eckert MA, Keren NI, Roberts DR, Calhoun VS \& Harris KC. Age-related changes in processing speed: unique contributions of cerebellar and prefrontal cortex. Frontiers in Human Neuroscience 2010410. (doi:10.3389/neuro.09.010.2010)

23 Schmahmann JD \& Sherman JC. The cerebellar cognitive affective syndrome. Brain 1998121 561-579. (doi:10.1093/brain/121.4.561)

24 Baldaçara L, Borgio JG, Lacerda AL \& Jackowski AP. Cerebellum and psychiatric disorders. Revista Brasileira de Psiquiatria 200830 281-289. (doi:10.1590/S1516-44462008000300016)

25 Bekkelund SI, Pierre-Jerome C, Husby G \& Mellgren SI. Quantitative cerebral MR in rheumatoid arthritis. AJNR. American Journal of Neuroradiology 199516 767-772.

26 Oldfield RC. The assessment and analysis of handedness: the Edinburgh inventory. Neuropsychologia 19719 97-113. (doi:10.1016/00283932(71)90067-4)

27 Manor B, Newton E, Abduljalil A \& Novak V. The relationship between brain volume and walking outcomes in older adults with and without diabetic peripheral neuropathy. Diabetes Care 201235 1907-1912. (doi:10.2337/dc11-2463)

28 Barahona MJ, Sucunza N, Resmini E, Fernández-Real JM, Ricart W, Moreno-Navarrete JM, Puig T, Farrerons J \& Webb SM. Persistent body fat mass and inflammatory marker increases after long-term cure of Cushing's syndrome. Journal of Clinical Endocrinology and Metabolism 200994 3365-3371. (doi:10.1210/jc.2009-0766)

29 Webb SM, Badia X, Barahona MJ, Colao A, Strasburger CJ, Tabarin A, van Aken MO, Pivonello R, Stalla G, Lamberts SW et al. Evaluation of health-related quality of life in patients with Cushing's syndrome with a new questionnaire. European Journal of Endocrinology 2008158 623-630. (doi:10.1530/EJE-07-0762)

30 Ségonne F, Dale AM, Busa E, Glessner M, Salat D, Hahn HK \& Fischl B. A hybrid approach to the skull stripping problem in MRI. NeuroImage 200422 1060-1075. (doi:10.1016/j.neuroimage.2004.03.032)

31 Fichl B, Salat DH, Busa E, Albert M, Dieterich M, Haselgrove C, van der Kouwe A, Killiany R, Kennedy D, Klaveness $\mathrm{S}$ et al. Whole brain segmentation: automated labeling of neuroanatomical structures in the human brain. Neuron 200233 341-355. (doi:10.1016/S08966273(02)00569-X)

32 Fichl B, Salat DH, van der Kouwe AJ, Makris N, Ségonne F, Quinn BT \& Dale AM. Sequence-independent segmentation of magnetic resonance images. NeuroImage 200423 (Suppl 1) S69-S84. (doi:10.1016/j.neuroimage.2004.07.016)

33 Andela CD, van der Werff SJ, Pannekoek JN, van den Berg SM, Meijer OC, van Buchem MA, Rombousts SA, van der Mast RC, Romijn JA, Tiemensma J et al. Smaller grey matter volumes in the anterior cingulate cortex and greater cerebellar volumes in patients with long-term remission of Cushing's disease: a case-control study. European Journal of Endocrinology 2013169 811-819. (doi:10.1530/EJE-13-0471)
34 Bhatt AJ, Feng Y, Wang J, Famuyide M \& Hersey K. Dexamethasone induces apoptosis of progenitor cells in the subventricular zone and dentate gyrus of developing rat brain. Journal of Neuroscience Research 201391 1191-1202. (doi:10.1002/jnr.23232)

35 Wang J, Barak LS, Mook RA Jr \& Chen W. Glucocorticoid hedgehog agonists in neurogenesis. Vitamins and Hormones 201187 207-215. (doi:10.1016/B978-0-12-386015-6.00030-5)

36 Tata DA, Marciano VA \& Anderson BJ. Synapse loss from chronically elevated glucocorticoids: relationship to neuropil volume and cell number in hippocampal area CA3. Journal of Comparative Neurology 2006498 363-374. (doi:10.1002/cne.21071)

37 Kleen JK, Sitomer MT, Killeen PR \& Conrad CD. Chronic stress impairs spatial memory and motivation for reward without disrupting motor ability and motivation to explore. Behavioral Neuroscience 2006120 842-851. (doi:10.1037/0735-7044.120.4.842)

38 Galvan V \& Jin K. Neurogenesis in the aging brain. Clinical Interventions in Aging 20072 605-610.

39 Balietti M, Fattoretti P, Giorgetti B, Casoli T, Di Stefano G, Platano D, Aicardi G, Lattanzio F \& Bertoni-Freddari C. Effect of two medium chain triglycerides-supplemented diets on synaptic morphology in the cerebellar cortex of late-adult rats. Microscopy Research and Technique 200972 933-938. (doi:10.1002/jemt.20737)

40 Lauterbach EC, Harris JB \& Bina WF III. Mood and neurobehavioral correlates of cerebellar lesions. Cognitive and Behavioral Neurology 2010 23 63-73. (doi:10.1097/WNN.0b013e3181cfb541)

41 Bauer PM, Hanson JL, Pierson RK, Davidson RJ \& Pollak SD. Cerebellar volume and cognitive functioning in children who experienced early deprivation. Biological Psychiatry 200966 1100-1106. (doi:10.1016/ j.biopsych.2009.06.014)

42 Resmini E. Persistent comorbidities in Cushing's syndrome after endocrine cure. Advances in Endocrinology 2014. (doi:10.1155/2014/231432)

43 Heringa SM, van den Berg E, Reijmer YD, Nijpels G, Stehouwer CG, Teerlink T, Scheffer PG, van den Hurk K, Kappelle LJ, Dekker JM et al. Markers of low-grade inflammation and endothelial dysfunction are related to reduced information processing speed and executive functioning in an older population - the Hoorn Study. Psychoneuroendocrinology 201440 108-118. (doi:10.1016/j.psyneuen.2013.11.011)

44 Gustand J, Brickman AM, Paul RH, Browndyke J, Moser DJ, Ott BR, Gordon N, Haque O \& Cohen RA. Progressive morphometric and cognitive changes in vascular dementia. Archives of Clinical Neuropsychology 200520 229-241. (doi:10.1016/j.acn.2004.07.001)

45 Scherder E. Functional circuits in cognitive aging and mild cognitive decline. In Aging and Dementia: Neuropsychology, Motor Skills, and Pain, ch 2, p 49. Amsterdam: VU University Press, 2011.

46 Dichgans J \& Mauritz KH. Patterns and mechanisms of postural instability in patients with cerebellar lesions. Advances in Neurology 198339 633-643.

47 Horak FB \& Diener HC. Cerebellar control of postural scaling and central set in stance. Journal of Neurophysiology 199472 479-493.

48 Singh D, Park W, Lewy MS \& Jung ES. The effects of obesity and standing time on postural sway during prolonged quiet standing. Ergonomics 200952 977-986. (doi:10.1080/00140130902777636)

49 Fjeldstad C, Fjeldstad AS, Acree LS, Nickel KJ \& Gardner AW. The influence of obesity on falls and quality of life. Dynamic Medicine 20087 4. (doi:10.1186/1476-5918-7-4)

50 Rubenstein LZ. Falls in older people: epidemiology, risk factors and strategies for prevention. Age and Ageing 200635 (Suppl 2) ii37-ii41. (doi:10.1093/ageing/afl084)

Received 9 May 2014

Revised version received 30 June 2014

Accepted 8 July 2014 